\title{
Diferencias en el uso del Papanicolau entre médicos de atención primaria
}

\author{
Diferences between Papanicolau use of primary care
}

\section{Objetivos}

Evaluar creencias y prácticas relacionadas al rastreo del cáncer de cuello uterino ( $\mathrm{CCU})$ en médicos de atención primaria.

\section{Diseño, población y área geográfica}

Estudio observacional de corte transversal realizado entre 2006 y 2007 sobre una muestra representativa de 471 médicos de familia, 310 clínicos y 333 gineco-obstetras de EE.UU.

\section{Medición de resultados principales}

Mediante una encuesta que utilizó casos clínicos como disparadores, se indagó sobre creencias, actitudes y prácticas en relación al rastreo de CCU, contrastando la consistencia de las respuestas con guías de práctica clínica (GPC) vigentes ${ }^{1-3}$.

\section{Resultados}

La tasa de respuesta fue del $67,5 \%$. En forma global, el $91 \%$ de los encuestados (IC95\% 89 a 92,6) reportó solicitar Pap a sus pacientes, variando según la especialidad, en la periodicidad, consistencia con las recomendaciones y creencias acerca del rastreo $(p<0,001)$. Si bien la mayoría de los solicitantes de Pap $(84,3 \%)$ reportó actuar muy influenciados por GPC, solo una minoría brindo recomendaciones completamente consistentes con las mismas en cuanto a inicio y finalización del ras- treo. Tras un análisis multivariado, los médicos de familia y clínicos mostraron mayor consistencia en el adecuado seguimiento de GPC que los ginecólogos (Tabla 1). En la mayoría de las respuestas se observo una tendencia a la sobreutilización del rastreo.

Tabla 1: Consistencia de las recomendaciones con las guías según especialidad

\begin{tabular}{l|c|c|c}
\multicolumn{1}{c|}{ Resultados } & $\begin{array}{c}\text { Sensibilidad } \\
\text { (IC95\%) }\end{array}$ & $\begin{array}{c}\text { Especificidad } \\
\text { (IC95\%) }\end{array}$ & $\begin{array}{c}\text { Médicos de familia } \\
(\mathbf{n = 4 7 1 )}\end{array}$ \\
\hline Consistencia & $16,4 \%$ & $27,5 \%$ & $21,1 \%$ \\
\hline OR ajustado (IC 95\%) & 1 & $1,98(1,22-3,23)$ & $1,45(0,99-2,13)$ \\
\hline
\end{tabular}

OR: odds ratio

\section{Conclusiones}

El rastreo que los médicos reportan no es consistente con las recomendaciones reflejando un sobre-utilización. Se requerirá la implementación de intervenciones efectivas que se enfoquen en la modificación de factores médicos y de la práctica para mejorar el rastreo del CCU.

Palabras claves: rastreo, cáncer de cuello, Papanicolaou.

Key words: screening, uterine cervical cancer, pap smear.

Fuente de financiamiento: Instituto Nacional del Cáncer.

\section{Comentario}

EI PAP constituye una práctica de rastreo fuertemente recomendada ${ }^{1-3}$ ya que disminuye la mortalidad por CCU. Su uso inadecuado puede deberse entre otros, a la falta de información en médicos o la sobre-prestación de la practica asociada a incentivos económicos. Esto último podría provocar un incremento en los $\operatorname{costos}^{4}$, en el seguimiento de pruebas innecesarias ${ }^{5}$, así como también generar preocupación en las pacientes.

La recomendación del rastreo por el médico de atención primaria cumple un rol central en la detección del $\mathrm{CCU}^{6}$. La insuficiente información o educación en las pacientes, puede llevar a que éstas perciban la sobre atención como algo positivo. Este estudio mostró una baja consistencia con las recomendaciones vigentes. Considerando que se trata de auto-reporte de profesionales motivados en responder hace probable que la práctica real sea aun peor.

Entre sociedades científicas existe controversia en la edad de discontinuación, la periodicidad del rastreo o la técnica a emplear. No hay estudios que evalúen la efectividad del rastreo en mujeres mayores de 70 años ${ }^{7}$ así como tampoco que determinen la frecuencia ideal. Sin embargo, los casos de CCU invasivos son más probables por fallas en el rastreo que por la técnica utilizada. Más de la mitad de mujeres que desarrollan CCU nunca recibieron un PAP, o lo hicieron en forma esporádica ${ }^{8}$.

\section{Conclusiones del comentador}

La educación en temas sexuales y ginecológicos en adolescentes y mujeres en edad sexual activa no debiera diferirse hasta el inicio del rastreo del CCU. La reducción de la prevalencia del CCU depende tanto de la educación del profesional como del paciente con el fin de extender la cobertura del rastreo, de definir criterios en las recomendaciones y de involucrar al paciente en la toma de decisiones.

lara Alonso [ Servicio de Medicina Familiar y Comunitaria, Hospital Italiano de Buenos Aires. iara.alonso@hospitalitaliano.org.ar ]

Alonso I. Diferencias en el uso del PAP entre médicos de atención primaria. Evid Act Práct Ambul. Jul-Set 2011;14(3):86. Comentado de: Yabroff KR y col. Specialty differences in primary care physician reports of papanicolaou test screening practices: a national survey, 2006 to 2007 . Ann Intern Med. 2009;151(9):602-11. PMID: 19884621.

\section{Referencias}

1. U.S. Preventive Services Task Force. Screening for cervical cancer: recommendations and rationale. Am J Nurs. 2003;103:101-2, 105-6, 108-9.

2. Saslow D, y col. American Cancer Society. American Cancer Society guideline for the early detection of cervical neoplasia and cancer. CA Cancer J Clin. 2002;52:342-62.

3. ACOG Committee on Practice Bulletins. ACOG Practice Bulletin: clinical management guidelines for obstetrician-gynecologists. Number 45, August 2003. Cervical cytology screening (replaces committee opinion 152, March 1995). Obstet

Gynecol. 2003;102:417-27

4. Helms LJ, y col. Determining costs of health care services for cost-effectiveness analyses: the case of cervical cancer prevention and treatment. Med Care. 1999; 37:652-61.

5. Stout NK, y col. Trade-offs in cervical cancer prevention: balancing benefits and risks. Arch Intern Med. 2008; 168:1881-9.

6. Coughlin SS, y col. Physician recommendation for Papanicolau testing among U.S. women, 2000. Cancer Epidemiol Biomarkers Prev. 2005;14:1143-8.

7. IARC Working Group on Cervical Cancer Screening. Summary Chapter. In: Hakama M, y col. Screening for cancer of the uterine cervix. IARC Scientic Publications No.76. IARC, Lyon 1986.

8. Abed Z, y col. Cervical screening history in patients with early stage carcinoma of the cervix. Ir Med J 2006; 100:140. 